\title{
Identificación de áreas potenciales para la implementación del sistema agroforestal Quesungual en el Valle del Cauca ${ }^{1}$
}

\author{
Identification of Potential Areas for the Implementation of the \\ Quesungual an Agroforestry System in the Valle del Cauca
}

\section{Jorge Eliecer Rubiano Mejía $^{2}$ \\ Mauricio Rincón Romero ${ }^{3}$ \\ Fabio Alexander Castro Llanos ${ }^{4}$}

Para citar este artículo, utilice el nombre completo así:

Rubiano, J., Rincón Romero, M. \& Castro Llanos, F. (2014). Identificación de áreas potenciales para la implementación del sistema agroforestal Quesungual en el Valle del Cauca. Perspectiva Geográfica, 19(2), 201-218

\section{Resumen}

Los sistemas agroforestales son una forma alternativa de uso de la tierra que combina componentes leñosos con cultivos o animales. El presente estudio identifica áreas con potencial para implementar el sistema agroforestal Quesungual (SAQ), una de las formas de uso de éxito reconocido en países de Centroamérica, en zonas de ladera del departamento del Valle del Cauca como insumo en el proceso de ordenamiento del territorio. Estos sistemas de manejo de la tierra son una alternativa productiva y sostenible para dichas regiones, porque simulan las características de los ecosistemas naturales en su estructura y función. El objetivo de este estudio es ubicar las zonas de mayor aptitud para desarrollar

\footnotetext{
1 Investigación presentada a la convocatoria interna de investigación de la Universidad del Valle para la conformación de un banco de proyectos - Ciencias sociales y humanas - Vicerrectoría de Investigaciones. Grupos de Investigación: Territorios Departamento de Geografía y GISMODEL- Grupo de Investigación en Simulación y Modelación Dinámica Espacial, de la Escuela de Ingeniería Civil y Geomática, Universidad del Valle.

2 PhD en Geografía. Profesor asociado, Universidad del Valle, Colombia. Correo: jorge.rubiano@correounivalle.edu.co

$3 \mathrm{PhD}$ en Geografía. Profesor asistente, Universidad del Valle, Colombia. Correo: mauricio.rincon@correounivalle.edu.co

4 Estudiante de Geografía. Universidad del Valle, Colombia. Correo: fabio.castro@correounivalle.edu.co
} 
el SAQ en el Valle del Cauca haciendo uso de las técnicas de análisis espacial, peso de la evidencia y regresión logística.

Los resultados generados son evaluados a la luz de pruebas estadísticas integradas dentro de las herramientas de análisis espacial y posteriormente socializados ante profesionales de las Unidades Municipales de Asistencia Técnica de tres municipios con mayor potencial. Los resultados permitieron ubicar con detalle las zonas de mayor aptitud, que suman alrededor de 232.000 hectáreas como aptas para el establecimiento del SAQ, lo cual podría ser utilizado en los planes de ordenamiento de municipios de la región.

Palabras clave: agroforestería, análisis espacial, bosque seco tropical, ordenamiento territorial.

\begin{abstract}
Agroforestry systems are an alternative form of land use that combines woody components with crops and/or animals. This study identifies potential areas to implement the agroforestry system known as the Quesungual (SAQ), one of such forms of use which has had recognized success in countries in Central America, on slopes of Valle del Cauca as input in the process of land management. These systems are a productive and sustainable alternative in these regions while simulating the characteristics of natural ecosystem structure and function. The aim of this study is to locate the areas of greatest potential to implement the SAQ in the Valle del Cauca using spatial analysis techniques such as the weights of evidence and logistic regression. The results were evaluated both with statistical techniques integrated within spatial analysis tools and afterwards through the knowledge shared with professionals from three Municipal Technical Assistance Units (UMATAS). The results allowed locating in detail the areas of highest fitness, which could be used in management plans for municipalities in the region, totaling around 232,000 hectares (ha) as suitable for the establishment of the SAQ.
\end{abstract}

Keywords: agroforestry, spatial analysis, land management, dry tropical forest. 


\section{Introducción}

Alrededor del $60 \%$ del territorio colombiano está conformado por paisajes de montaña y lomerío (Malagón, 2003). De estos, menos del $5 \%$ conserva su vegetación natural, más del $70 \%$ está dedicado a la ganadería y cerca del $25 \%$ a cultivos transitorios, permanentes y heterogéneos (IDEAM, 2010). Estos sistemas de producción se caracterizan por el uso de prácticas insostenibles en el manejo de los suelos, lo cual ha conducido al deterioro de su calidad, reflejado en la erosión y degradación de los mismos. Parte de la solución para enfrentar esta situación está en manos de las autoridades municipales, las cuales están en la obligación de implementar procesos de ordenamiento territorial que acerquen el uso del suelo con su vocación. El establecimiento de sistemas agroforestales (SA) se constituye como una propuesta tecnológica estratégica para ser implementada por los municipios del país desde los planes de ordenamiento territorial (POT), con lo que se esperaría reducir y quizás eliminar los efectos adversos de los sistemas agrícolas actuales (Organización de las Naciones Unidas para la Alimentación y la Agricultura -FAO-, 2014).

Existen experiencias en países vecinos donde han probado sus beneficios, como ocurre en Centroamérica, particularmente en Honduras y Nicaragua con el SAQ. Dicho sistema ha permitido la integración espontánea de árboles dispersos en número significativo $\mathrm{y}$ en medio de zonas de cultivo o pastos, y de los cuales se utilizan podas periódicas para incrementar el aporte de materia orgánica al suelo.

En esta dirección, el presente estudio tuvo como objetivo identificar, por medio de técnicas de análisis espacial, áreas con potencial para implementar el sistema agroforestal Quesungual, como alternativa al manejo actual en zonas de ladera que presenten procesos de degradación en el departamento del Valle del Cauca.

Para el logro de dicho objetivo se seleccionó una experiencia considerada exitosa a nivel regional, que tiene lugar en los países de Honduras y Nicaragua, en los cuales se han puesto en marcha sistemas agroforestales en regiones similares a las del Valle del Cauca en los aspectos social y ambiental. Tal es el caso de la región de Lempira en Honduras, la cual está ubicada en el corredor del bosque seco tropical centroamericano, similar a los relictos de bosque seco encontrados en Colombia, localizados en la llanura del Caribe, y los valles interandinos de los ríos Cauca y Magdalena, clasificados dentro de este ecosistema. Según el Instituto de Investigación de Recursos Biológicos 
Alexander von Humboldt, el bosque seco tropical ha sido considerado estratégico en Colombia para implementar acciones de conservación y de ordenamiento, teniendo en cuenta que pocas áreas de este ecosistema se encuentran bajo régimen de protección (Instituto de Investigación de Recursos Biológicos Alexander von Humboldt, 2014).

\section{Ordenamiento territorial}

El ordenamiento territorial es entendido como el conjunto de acciones concertadas, diseñadas para orientar la transformación, ocupación y utilización de los espacios geográficos buscando su desarrollo socioeconómico, teniendo en cuenta las necesidades e intereses de la población, las potencialidades del territorio y considerando la armonía con el medio ambiente (Ley 388 de 1997). Producto de este proceso surgen los POT que desde el año 2010 vienen siendo objeto de evaluación y ajuste. Se ha evidenciado que los POT, a pesar de los avances logrados, aún carecen de propuestas a nivel local, entre las que se cuenten alternativas de manejo para el territorio objeto de análisis (Carrión, 2008). En consecuencia, los POT hoy se ven abocados a mejorar el análisis de los datos territoriales integrando el contexto biofísico y social con el potencial de provisión de servicios ecosistémicos y ambientales disponibles en las unidades territoriales, como son los municipios y las cuencas. Se hace necesario desde esta perspectiva abordar la carencia de propuestas tecnológicas para regiones particulares con propuestas concretas de manejo de las mismas.

\section{Sistemas agroforestales}

Los sistemas agroforestales surgen como estrategias de manejo adaptables a unos rangos amplios de condiciones ambientales y socioeconómicas. Los sistemas agroforestales son asociaciones de cultivos agrícolas anuales o semestrales con plantas perennes forestales $\mathrm{o}$ frutales que favorecen el aprovechamiento de nutrientes y agua, puesto que contribuyen a la conservación y mantenimiento de la calidad y fertilidad de los suelos (Terán \& Vidal, 2013). Estos sistemas han sido concebidos desde el enfoque de producción, como una réplica de los sistemas naturales, en los cuales la diversidad de especies en tiempo y espacio es la estrategia para el logro de la sostenibilidad del conjunto. Entre los diversos servicios que prestan los sistemas agroforestales, además de los productos agrícolas y forestales, se incluyen la conservación de suelos y aguas, mantenimiento de la biodiversidad, mayor resiliencia de las unidades productivas a efectos climáticos y de mercado adversos al sistema, y como estrategia de diversificación por 
parte de los productores rurales en la generación de ingreso y seguridad alimentaria. Estos beneficios se han demostrado en las laderas del sur de Honduras en Centroamérica.

\section{El Sistema Agroforestal Quesun- gual}

En el año de 1992, en el marco de la ejecución del Proyecto Desarrollo Rural del Sur de Lempira, se observó en algunas comunidades la existencia de una práctica agrícola muy generalizada entre los productores, que consistía en podar sus árboles para sembrar frijol y maíz. El producto de la poda se acumulaba sobre el terreno y posteriormente se incorporaba esta materia orgánica como abono para el cultivo. Tal técnica, al parecer, se trata de una práctica ancestral heredada de los campesinos oriundos de Lempira, denominado actualmente sistema agroforestal "Quesungual" (SAQ) (Food and Agricultural Organization -FAO-, Programa para la Agricultura Sostenible en Laderas de América Central -PASOLAC-, Instituto Nacional de Investigación Agraria y Alimentaria -INIA- \& Servicio Agrícola Ganadero -SAG-, 2005). Tal sistema integra el componente arbóreo dentro de las unidades agrícolas como un elemento estratégico para la conservación de los suelos, aumentando la retención de humedad de los mismos, mediante las sucesivas adiciones a la tierra de materia orgánica proveniente del follaje (Pauli, Barrios, Conacher \& Oberthür, 2011, 2012). El SAQ integra el componente arbóreo en las fincas de manera dispersa, con lo que se favorece producir en un mismo lote, no solo leña y madera derivadas del manejo de la regeneración natural, sino también el cultivo de granos para alimentación familiar y venta. Los beneficios adicionales incluyen la reducción de la vulnerabilidad física y social de las familias, mitigación de los efectos del cambio climático, reducción de la degradación de tierras, la desertificación y los riesgos para la seguridad alimentaria. Hoy en día, luego de más de dos décadas de implementación del SAQ, se reconocen los beneficios evidenciados en el mejoramiento de los ingresos, la calidad de vida de la población local y en un mejor estado de los suelos y aguas donde este se ha desarrollado (Pauli et al., 2012).

\section{Metodología}

El enfoque utilizado en este estudio es conocido con el nombre de dominios de extrapolación (Rubiano \& Soto, 2009). Esta es una metodología para el manejo de datos geográficos utilizada en la identificación de sitios similares o "dominios de extrapoblación”, dadas unas características de tipo biofísico y socioeconómico particulares y de relevancia para el 
fenómeno en estudio. Su uso tiene potencial en un rango amplio de actividades donde se requiere la selección de un lugar o la realización de análisis espacial para la comprensión de fenómenos de diversa índole.

La metodología tiene su origen en la exploración geológica (BonhamCarter \& Agterberg, 1990; Porwal, González-Álvarez, Markwitz, McCuaig \& Mamuse, 2010), pero ha sido igualmente utilizada con éxito en ciencias médicas (Spiegelhalter, 2009) y en estudios arqueológicos (Duke \& Steele, 2010), entre otros. La metodología se basa en el uso de dos técnicas estadísticas adaptadas al análisis espacial e incorporado en sistemas de información geográfica (SIG): la regresión logística y el peso de la evidencia. Ambas técnicas se utilizan para generar superficies de probabilidad de la ocurrencia de eventos de los cuales se conoce poca o limitada información. Los modelos probabilísticos de regresión logística y peso de la evidencia son utilizados para el cálculo de la probabilidad condicional. Los resultados se acompañan del cálculo de incertidumbre o confianza en cada valor de probabilidad, el cual es una medida de la varianza en la estimación del valor de probabilidad (Agterberg, Bonham-Carter \& Wright, 1990). La estimación del valor de probabilidad puede ser mejorada con la incorporación de nueva información sobre el evento, a través de la observación directa en campo o por medio de comparaciones entre los resultados obtenidos en los SIG y observación directas en zonas de alta probabilidad. Las técnicas mencionadas permiten la incorporación de información capturada sistemáticamente por sensores, encuestas o por la observación u opinión de expertos.

\section{1 Área de estudio}

El área de estudio incluyó, además de la región de Lempira Sur, en donde tiene ocurrencia del SAQ, todo el departamento del Valle del Cauca, en el cual se realizó la estimación de las áreas potenciales (Figura 1).

\subsection{Selección y descripción de las variables}

Para la estimación de la probabilidad de similitud con los sitios donde ocurre el evento de interés, en este caso el SAQ, fue necesario el uso de un grupo de variables clave o variables de evidencia basadas en características espaciales y de contexto del SAQ (Tabla 1). La selección de dichas variables se realizó con la colaboración de expertos en el estudio y manejo del SAQ, los cuales fueron consultados vía electrónica.

Teniendo en cuenta el acceso limitado y la baja disponibilidad de información 

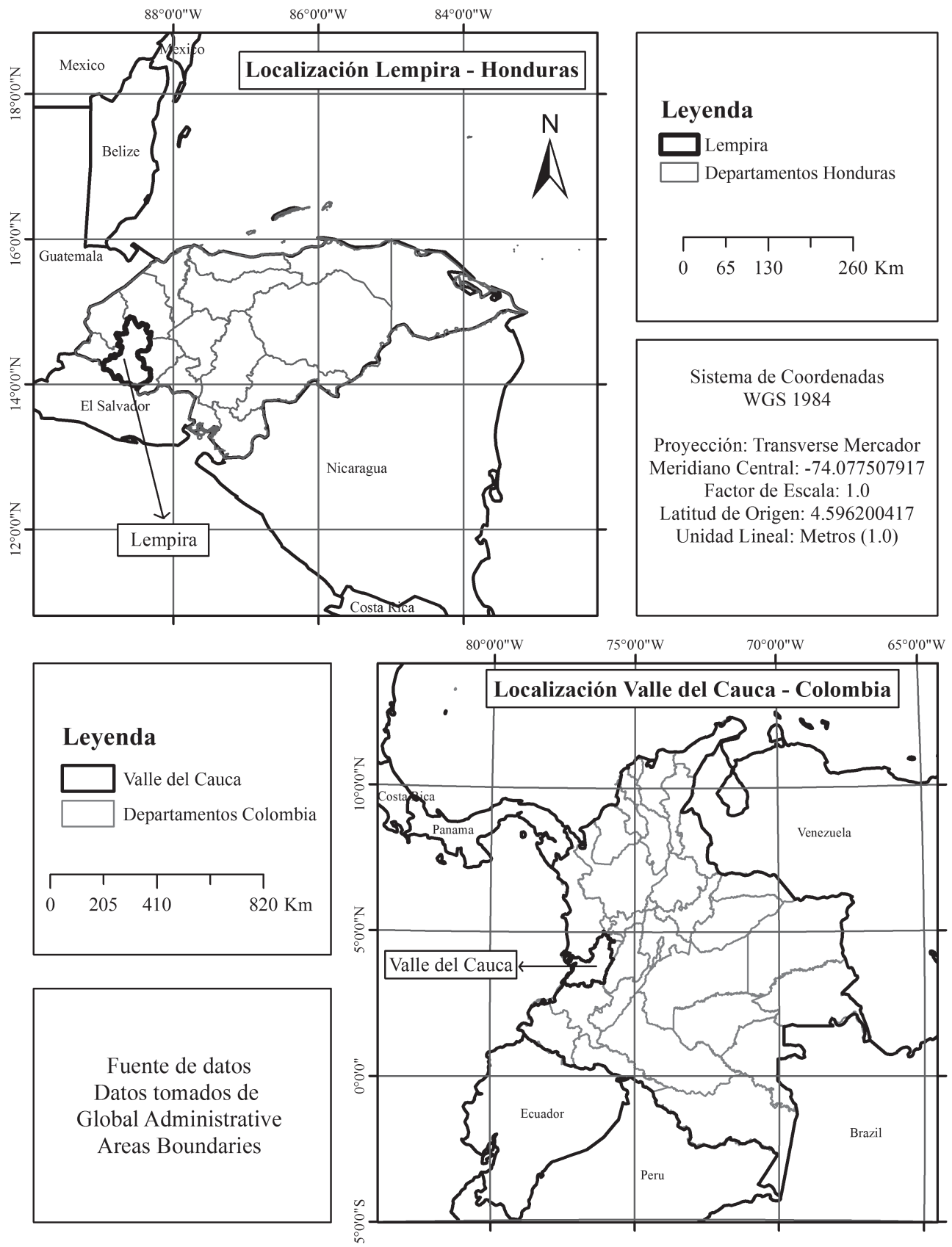

Figura 1. Área de estudio

Fuente: Datos tomado de Global Administrative Areas Boundaries. Del sitio de origen en Honduras se utilizó la localización de 218 unidades productivas con presencia del SAQ, georreferenciadas en el año de 2001 y suministradas en un archivo de puntos por el Centro Internacional de Agricultura Tropical (CIAT). 
de fuentes nacionales, así como la falta de compatibilidad con fuentes de información de países de la región, fue necesario hacer uso de bases de datos globales de tipo geográfico de libre acceso, provenientes de fuentes de alta confiabilidad, disponibles a 1 $\mathrm{km}$ de resolución. La implementación de esta información redujo los costos y garantizó un grado aceptable de certidumbre en cuanto a la calidad de la información generada.

Tabla 1. Listado de variables sugeridas para los dominios de extrapolación

\begin{tabular}{l}
\hline \multicolumn{1}{c}{ Variable de evidencia } \\
\hline Accesibilidad \\
Árbol de hoja ancha \\
Rastrojos-vegetación dispersa \\
Área agrícola \\
Pendiente del terreno \\
Índice topográfico de humedad \\
\hline
\end{tabular}

Fuente: elaboración propia.

La variable de accesibilidad identifica la distancia de cualquier lugar de la superficie de la tierra a centros poblacionales de más de 50000 habitantes. La metodología implementada para su cálculo se basó en la descrita por Uchida et al. (2009). Dicha variable conjuga aspectos tales como proximidad a los mercados, acceso a vías de comunicación, grado de acceso a servicios e infraestructura, barreras físicas como la pendiente, entre otros; todos estos importantes en la configuración de los sistemas de producción. Los tipos de vegetación, como los bosques de hoja ancha, los herbazales y los rastrojos fueron definidos con base en mapas de cobertura de la tierra disponibles para el año 2009 a nivel global. Estas categorías indican la presencia del recurso forestal y las actividades asociadas a este, además de las actividades agrícolas y ganaderas, las cuales fueron consideradas prerrequisito para el establecimiento de sistemas agroforestales. La pendiente del terreno igualmente es un aspecto esencial en la definición de áreas potenciales, ya que el SAQ es propio de los cultivos de subsistencia ubicados en la zona de ladera de Lempira Sur (Pauli et al., 2011). El índice topográfico de humedad se utilizó como una característica en la composición del suelo, que ayuda a determinar el establecimiento del SAQ según la disponibilidad potencial de agua para los cultivos. Esta característica, al igual que la pendiente del terreno, fue determinada a partir del modelo de elevación digital del terreno obtenido de bases de datos de correcciones del Shuttle Radar Topographic Mission (SRTM) (Jarvis et al., 2004).

\subsection{Procesamiento de los datos}

El conjunto de variables arriba descritas fue organizado dentro de un sistema de información geográfico 
(ArcGis v.9.3). Las herramientas estadísticas mencionadas están incorporadas en la extensión de libre acceso acoplable a este SIG, conocida como Spatial Data Modeller (Sawatzky, Raines \& BonhamCarter, 2009). Al interior de este se cuenta con la rutina de peso de la evidencia y regresión logística usados en el presente estudio. El protocolo para su uso demanda una preparación de las capas geográficas que describen las variables relacionadas con el sistema de proyección y coordenadas rutinarias para la integración de datos geográficos. Las variables continuas fueron reclasificadas en un número suficiente de clases organizadas en rangos para permitir la distribución de los 218 puntos de los sitios con SAQ identificados previamente en Honduras. Estos puntos son considerados como los sitios de entrenamiento del modelo; la forma como se distribuyen los puntos en dichas clases se utiliza para indicar de manera cuantitativa la importancia positiva o negativa de la variable en relación con la presencia del SAQ.

De igual manera, las variables categóricas, como la cobertura de la tierra, fueron separadas en capas binarias que representaban la presencia o ausencia de cada cobertura. Las zonas sin presencia de la cobertura fueron llenadas con franjas distantes del uso en cuestión, dispuestas cada 1000 metros a la manera de corredores o "buffers". De esta manera se generaron superficies que cubrieron el $100 \%$ del área de estudio. Dichas variables alimentaron el sistema que, en conjunto con los sitios de entrenamiento $\mathrm{y}$ las rutinas estadísticas mencionadas, permitió la elaboración de los mapas de probabilidad potencial. La caja de herramientas que integra estas técnicas en el sistema de información geográfico contiene la prueba estadística de Agterberg y Cheng (Agterberg \& Cheng, 2002), la cual permite identificar problemas de redundancia en el uso de variables y la independencia entre las mismas. Es necesario realizar este procedimiento un número considerable de veces, hasta encontrar una configuración del modelo que cumpla con las pruebas de condicionalidad mencionadas. Finalmente, con la respuesta o modelo mejor adaptado a los requisitos de las pruebas, se procede a realizar la cartografía de los sitios similares o dominios de extrapolación, indicados por la probabilidad de semejanza con los lugares utilizados como entrenamiento, es decir, producto de la combinación de las variables utilizadas en la búsqueda de sitios similares a aquellos en donde el SAQ ya existe, se genera una superficie de probabilidad de similaridad con dichos sitios. Este mapa es el utilizado para prescribir el SAQ donde quiera que la probabilidad es alta. 
En la cartografía final adicionalmente se identifican aquellas zonas que poseen restricciones para el uso agrícola, como las áreas del Registro Único Nacional de Áreas Protegidas (RUNAP) y las Reservas Naturales de la Sociedad Civil (RNSC), con el fin de excluirlas de las zonas potenciales para el SAQ. Por medio del SIG se cuantificaron por municipio las áreas que tienen mayor área o proporción de su área potencial para el SAQ, con el objeto de listar aquellos que, por dichos criterios, pueden ser objeto de consideración en planes de diseminación y adopción del SAQ.

\subsection{Socialización de los resultados}

Una vez generada la cartografía y los demás resultados, se visitaron tres Unidades Municipales de Asistencia Técnica (UMATA) para socializar la información, con el fin de sondear la percepción de los técnicos de dichas unidades y obtener comentarios a la luz de la experiencia local sobre la conveniencia de la propuesta en el municipio. Para tal fin se efectuaron entrevistas semiestructuradas sobre las alternativas tecnológicas usadas en el manejo de las zonas de ladera en el municipio, la erosión y la degradación de tierras; adicionalmente se hicieron preguntas acerca de los sistemas de cultivo actuales y potenciales $\mathrm{y}$ los vínculos entre la UMATA y la Oficina de Planeación en relación con el ordenamiento territorial. Esta socialización tuvo como objetivo principal compartir un conocimiento que puede ser utilizado para orientar el accionar de dichas unidades en apoyo a los planes de ordenamiento del territorio.

\section{Resultados}

El modelo que finalmente se utilizó para generar las áreas potenciales para el establecimiento del SAQ, se formula de la siguiente manera:

En este modelo se utilizaron como variables determinantes la presencia de cobertura con árboles de hoja ancha y las áreas con cobertura agrícola; en segunda instancia se tuvieron en cuenta el gradiente de la pendiente del terreno y la accesibilidad. Con relativa poca influencia, pero negativa, se incluyó el índice de humedad del terreno. Las zonas más húmedas no suelen usarse en el SAQ, pues están restringidas a sitios cercanos a cauces de ríos $\mathrm{y}$ vertientes, que coinciden con aquellas áreas de alta humedad en el suelo. La cobertura de rastrojos y vegetación dispersa fue excluida del modelo por presentar colinealidad con las otras coberturas.

La localización y distribución de las zonas con mayor y menor potencial para la implementación del SAQ, dadas las características incorporadas en el modelo de 
dominios de extrapolación dentro del similitud, se presentan en la Figura 2. departamento del Valle del Cauca Tal potencial se enuncia en el mapa y expresadas en probabilidad de

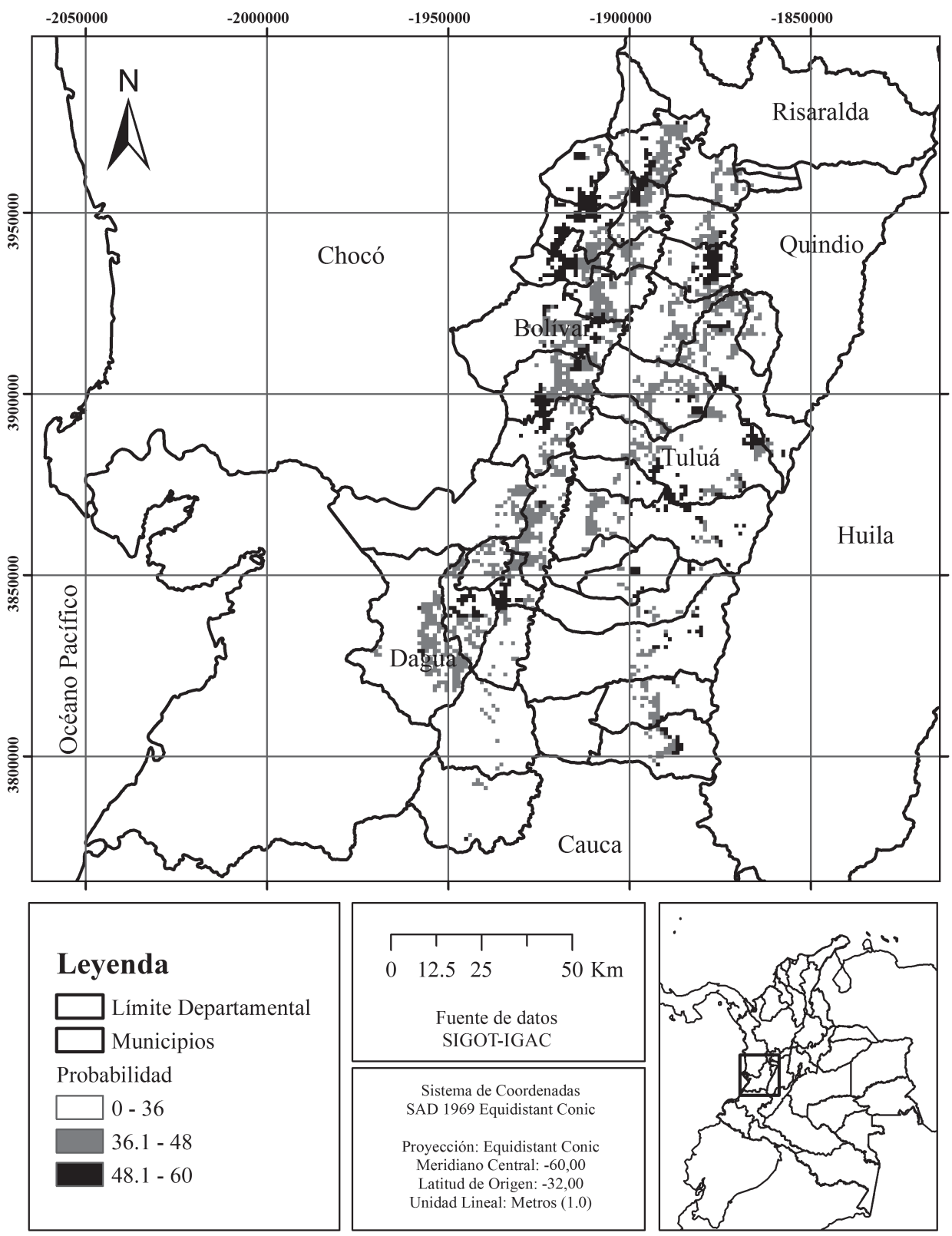

Figura 2. Áreas con aptitud para la implementación del SAQ en el Valle del Cauca Fuente: SIGOT, IGAC y datos de los autores. 
como porcentaje, significando que a mayor porcentaje hay mayor probabilidad o "aptitud" del área para implementar el SAQ. Las zonas en tonos grises y negros corresponden a aquellas con probabilidades entre un 36 $\%$ y un $60 \%$ de similitud con los sitios de origen del sistema en Honduras; las áreas en blanco representan zonas con muy baja probabilidad. Se observa que la mayoría de las áreas con probabilidades superiores al 36 $\%$ se encuentran a lo largo de las cordilleras que cruzan de sur a norte el departamento, pero en particular en la cordillera Occidental, mientras que las áreas más extensas se encuentran en el norte del departamento.
El valor de la confianza en la estimación de la probabilidad, por lo general, fue elevada, donde los valores de probabilidad fueron altos, presentándose algunas excepciones, como se observa en la Figura 4.

Los municipios con mayor área considerada apta para establecer sistemas agroforestales tipo Quesungual, son Tuluá, Bolívar y Dagua (Tabla 2). Es importante resaltar que el municipio de Dagua tiene dentro de su jurisdicción 3.888 ha consideradas reserva, las cuales fueron excluidas en la representación cartográfica.

Tabla 2. Primeros diez municipios con mayor área disponible y apta para el estableciiento del SAQ

\begin{tabular}{lcc}
\hline Municipio & $\begin{array}{c}\text { Hectáreas con } \\
\text { aptitud alta } \\
\text { X 1000 }\end{array}$ & $\begin{array}{c}\text { Área total municipal } \\
\text { ha X 1000 }\end{array}$ \\
\hline Tuluá & 14.3 & 818 \\
Bolívar & 13.7 & 815 \\
Dagua & 13.5 & 939 \\
Yotoco & 11.5 & 390 \\
Sevilla & 11.0 & 639 \\
Trujillo & 10.8 & 232 \\
Bugalagrande & 9.5 & 429 \\
Roldanillo & 8.4 & 209 \\
Ansermanuevo & 8.3 & 361 \\
El Dovio & 8.1 & 297 \\
\hline
\end{tabular}

Fuente: elaboración propia.

Los municipios con mayor aptitud para el establecimiento de SAQ, según su proporción de área municipal, son Vijes, Toro y Trujillo, los cuales tienen una característica en común al encontrarse en la vertiente 
oriental de la cordillera Occidental. La Figura 2 describe la proporción de para los diez primeros municipios del las áreas municipales en porcentaje

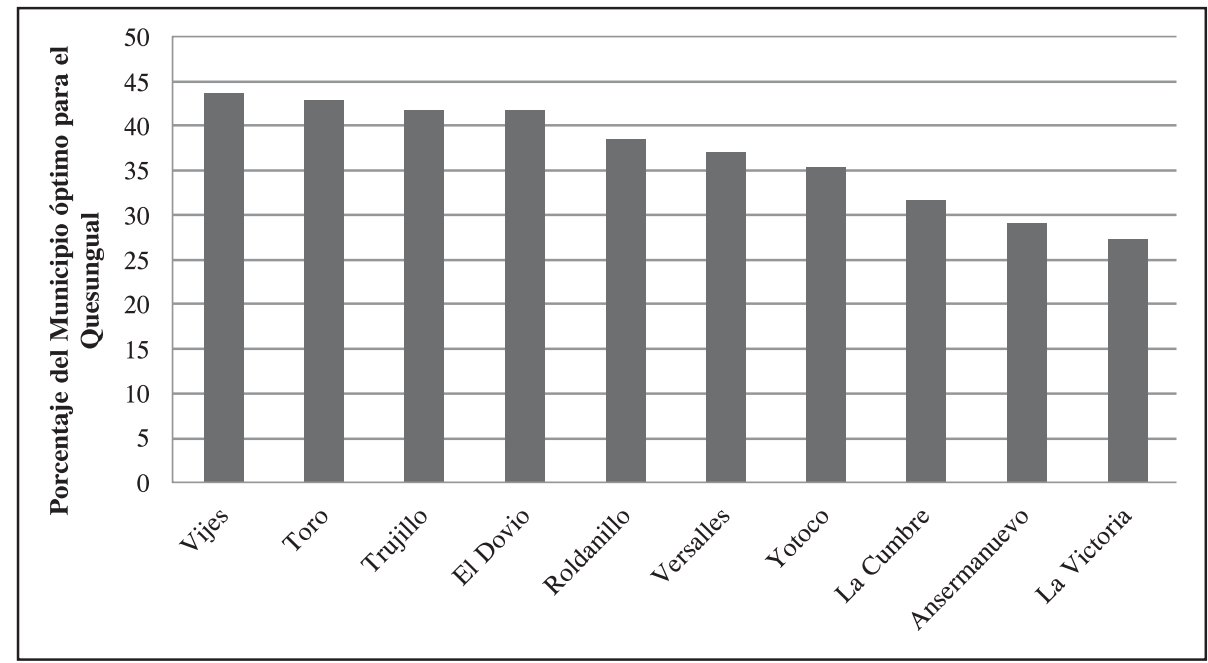

Figura 3. Municipios del Valle del Cauca con mayor aptitud para el SAF Quesungual en proporción al área municipal

Fuente: elaboración propia.

Con base en la información generada con los modelos probabilísticos, se visitaron las UMATA de los municipios de Toro, Tuluá y Dagua, para socializar el conocimiento generado y ponerlo al escrutinio de expertos y conocedores de la zona. La selección de estos municipios se hizo buscando aquellos con áreas representativas tanto en área total como proporcional al municipio $\mathrm{y}$ en función de la disponibilidad para atender nuestra visita. A manera de ejemplo, en el municipio de Toro se identificaron alrededor de 7.000 ha ubicadas en zonas sugeridas por el modelo. La Figura 5 presenta dichas áreas diferenciadas como cuadrículas por la resolución de pixel de $1 \mathrm{~km}$ de los datos fuente. Se observa que los corregimientos en el interior de dicho municipio y con mayor aptitud para el SAQ son La Pradera, San Francisco y Venta Quemada. Similar cartografía se presentó ante técnicos de los municipios de Tuluá y Dagua. La información y los datos obtenidos se consideraron muy orientadores y precisos según la opinión de los técnicos de las tres UMATA. También se reconoció la pertinencia de los sistemas agroforestales dentro de las estrategias de trabajo en estos municipios y la necesidad de 


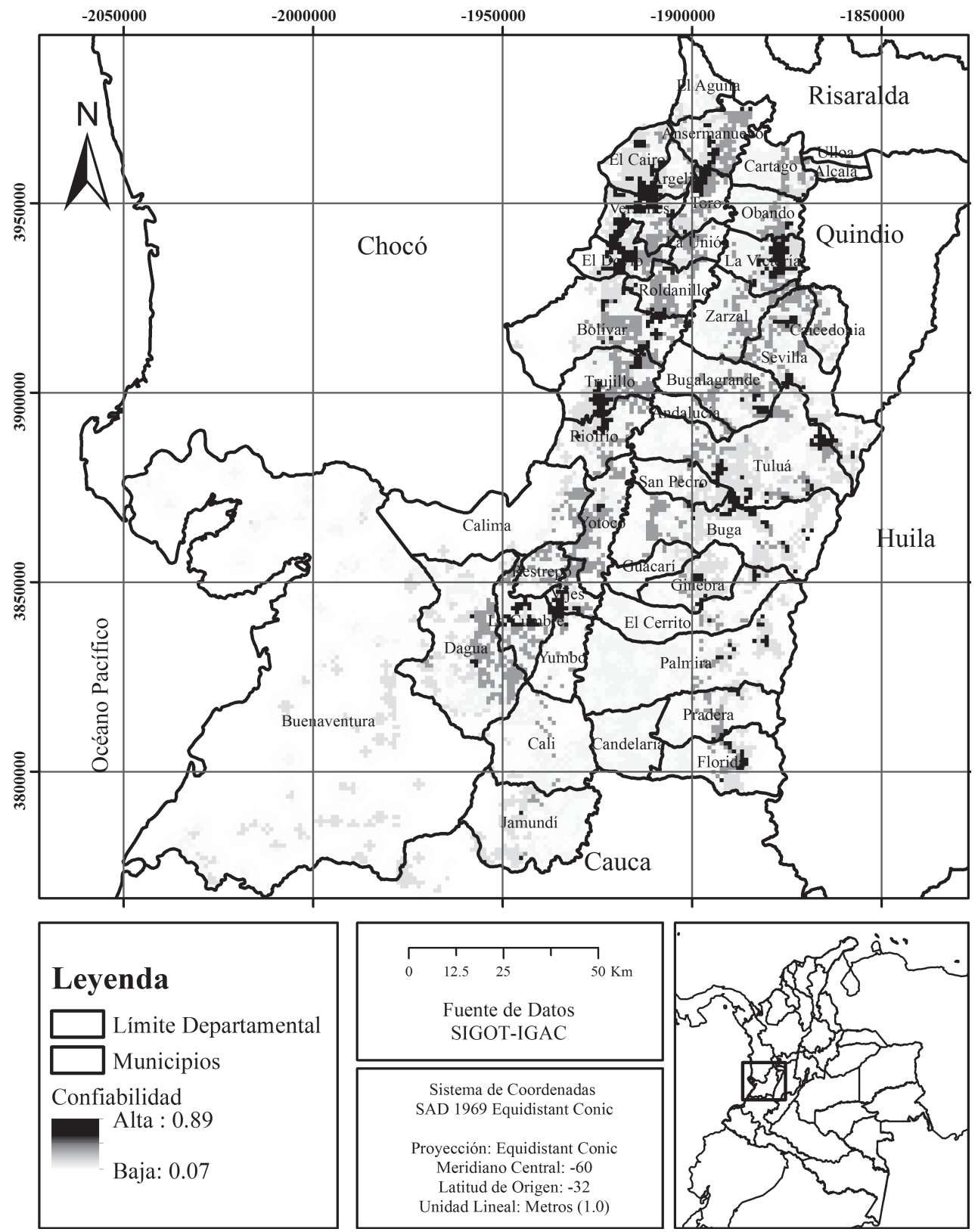

Figura 4. Confiabilidad en los valores de probabilidad

Fuente: SIGOT-IGAC y datos de los autores.

información de carácter espacial en particularparaelcálculodeinversiones la prescripción de los mismos, en en campo, a la hora de concebir un 
proyecto de implementación de SAQ en áreas definidas en los municipios. Los resultados socializados ante integrantes de las UMATA generaron gran expectativa en cuanto a su incorporación dentro de los planes de ordenamiento municipal, ya que estos obedecen a criterios integrales de valoración del potencial del territorio, al incluir variables de tipo biofísico y socioeconómico. La Figura 5 presenta el valor de confianza en la estimación de la probabilidad, la cual oscila entre cero y uno.

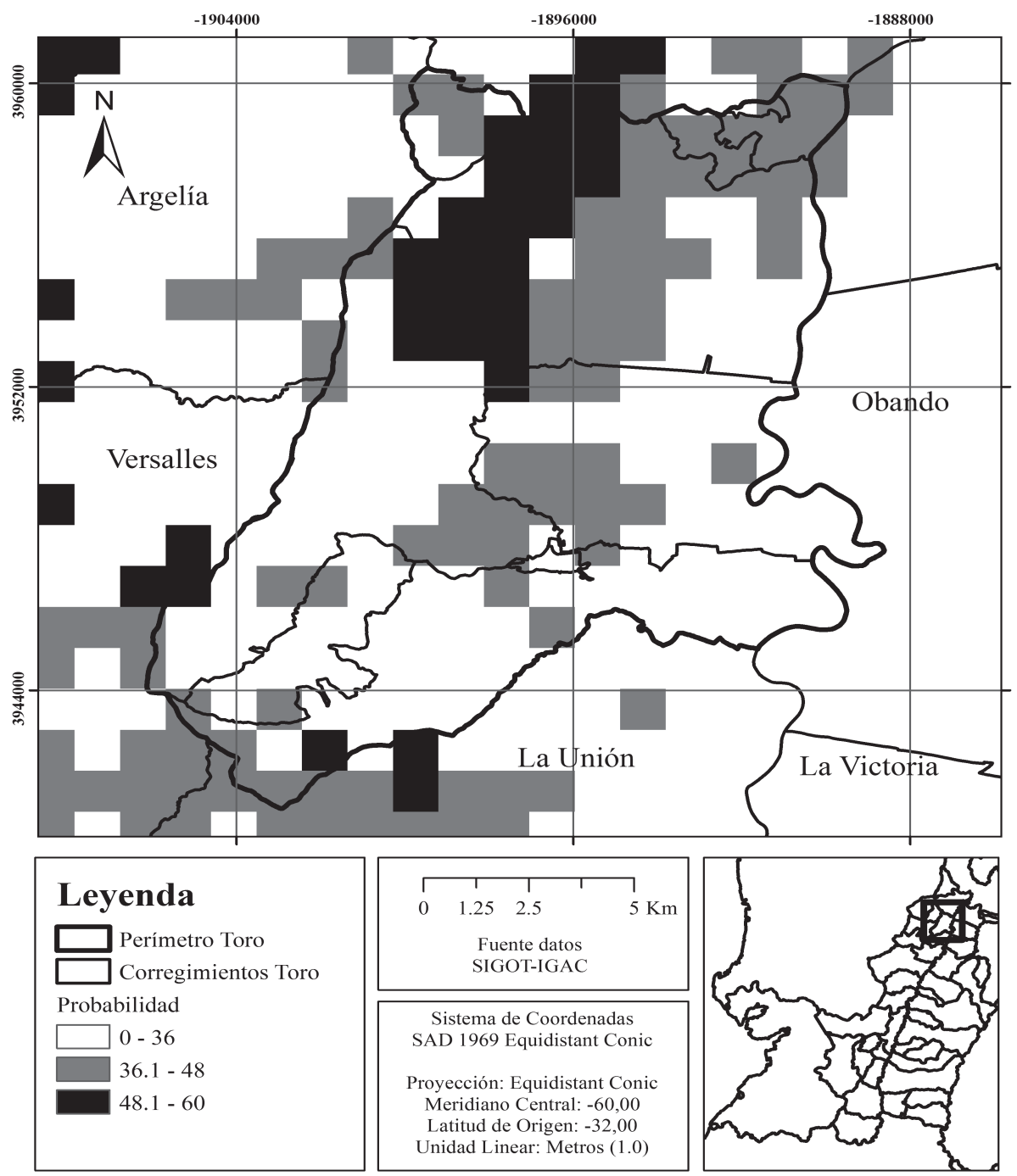

Figura 5. Áreas aptas por corregimiento para el SAQ en el municipio de Toro, Valle del Cauca Fuente: SIGOT-IGAC y datos de los autores. 


\section{Conclusiones y recomendaciones}

Áreas similares a aquellas de donde es originario el SAQ fueron identificadas en municipios del centro y norte del Valle del Cauca en Colombia. Los valores de probabilidad en su similitud con dichas zonas estuvieron por encima del $50 \%$, es decir que existe un margen amplio para que dichas áreas sean aptas para la implementación del sistema. Los municipios con mayores áreas consideradas como aptas para el SAQ fueron Tuluá, Bolívar y Dagua, los cuales suman en conjunto un total de 41.000 ha.

Las áreas identificadas con valores altos de probabilidad para el SAQ están contenidas en su mayoría dentro del ecosistema de bosque seco tropical del Valle del Cauca, lo que las convierte en zonas potenciales para iniciar procesos de restauración ecológica con actividades productivas correspondientes con condiciones socioeconómicas y ambientales similares a aquellas donde los SAQ han probado ser exitosos.

Entrevistas a técnicos de campo de municipios con áreas significativas consideradas con alta probabilidad, permitieron confirmar de manera cualitativa la pertinencia de los SAQ dentro de los municipios identificados con el modelo de dominios de extrapolación.
El método utilizado permitió incorporar información de diferentes tipos de datos, biofísicos y socioeconómicos, lo que facilitó un análisis más integral de prescripción del uso de la tierra en comparación con métodos tradicionales de designación del uso del suelo. Dado lo sistemático del método, es posible replicar la búsqueda de lugares potenciales en otros departamentos que enfrenten deterioro en sus zonas de ladera, como en el caso de los departamentos de Cauca y Nariño en Colombia. Igual aplica al evento en el cual se requiera identificar áreas para otros tipos de uso, como conservación de fuentes de agua o zonas para cultivos intensivos.

\section{Agradecimientos}

Esta investigación fue posible gracias a la financiación de la Vicerrectoría de Investigaciones de la Universidad del Valle y a la colaboración de técnicos de las Unidades Municipales de Asistencia Técnica Agropecuaria de los municipios de Toro, Tuluá y Dagua, y al CIAT por el suministro de los sitios georreferenciados de Honduras donde tiene lugar el SAQ. 


\section{Referencias}

Agterberg, F. \& Cheng, Q. (2002, Dec.). Conditional independence test for weights of evidence modeling. Natural Resources Research, 11(4), 249-255.

Agterberg, F.P., Bonham-Carter, G.F, \& Wright, D.F. (1990). Statistical pattern integration for mineral exploration. In G. Gaal \& D.F. Merriam (eds.). Computer applications in resource estimation: predictions and assessment for metals and petroleum. (pp. 1-21). Oxford: Pergamon.

Bonham-Carter, G.F. \& Agterberg, F.P. (1990). Application of a microcomputer based geographic information system to mineral-potential mapping. In J.T. Hanley, \& D.F. Merriam (eds.), Microcomputer-based applications in Geology, II, Petroleum. (pp. 49-74). New York: Pergamon Press.

Carrión G. B. A. (2008, junio). Debilidades del nivel regional en el ordenamiento territorial colombiano. Aproximación desde la normatividad política administrativa y de usos del suelo. Arquitectura, Ciudad y Entorno, 3(7), 145- 166.

Congreso de la República de Colombia. (1997). Ley 388 de 1997: por la cual se modifica la Ley 9 de 1989, y la Ley 2 de 1991 y se dictan otras disposiciones. Recuperado de http:// www.alcaldiabogota.gov.co/sisjur/normas/Norma1.jsp?i=339.

Duke, C. \& Steele, J. (2010, April). Geology and lithic procurement in Upper Palaeolithic Europe: a weights-of-evidence based GIS model of lithic resource potential, Journal of Archaeological Science, 37(4), 813-824.

IDEAM. (2010). Leyenda Nacional de Coberturas de la Tierra. Metodología CORINE Land Cover adaptada para Colombia Escala 1:100.000. Instituto de Hidrología, Meteorología y Estudios Ambientales. Bogotá, D. C., 72p

Instituto de Investigación de Recursos Biológicos Alexander von Humboldt. (2014). Bosques secos tropicales en Colombia. Recuperado de http://www.humboldt.org.co/ investigacion/ecosistemas-estrategicos/bosque-seco

Food and Agricultural Organization - FAO-, Programa para la Agricultura Sostenible en Laderas de América Central -PASOLAC-, Instituto Nacional de Investigación Agraria y Alimentaria - INIA- \& Servicio Agrícola Ganadero - SAG- (2005). Tecnologías y metodologías validadas para mejorar la seguridad alimentaria en las zonas secas de Honduras. Serie tecnologías: Manejo de suelos y agua. En B. Bustamante (comp.). (p. 107). Tegucigalpa: PESA/PASOLAC. Recuperado de http://www.pesacentroamerica. org/biblioteca/doc-honfeb/agua_suelos.pdf.

Malagón, C. D. (2003). Ensayo sobre tipología de suelos colombianos - Énfasis en génesis y aspectos ambientales. Rev. de la Academia Col. de Ciencias Exactas. Físicas y Naturales. XXVII(104), 319-341.

Jarvis, A. Rubiano, J., Nelson, A. Farrow, A. \& Mulligan, M. (2004). Practical use of SRTM data in the tropics, comparison with digital elevation models generated from cartographic data. Working Data, 198, 32.

Organización de las Naciones Unidas para la Alimentación y la Agricultura -FAO-. (2014). Políticas agroambientales en América Latina y el Caribe, Análisis de casos: Brasil, Chile, Colombia, México y Nicaragua. Santiago de Chile: FAO. 
Pauli, N., Barrios, E., Conacher, A.J. \& Oberthür, T. (2011). Soil macrofauna in agricultural landscapes dominated by the Quesungual Slash-and-Mulch Agroforestry System, western Honduras. Applied Soils Ecology, 47(2), 119-132.

Pauli, N., Barrios, E., Conacher, A.J. \& Oberthür, T. (2012). Farmer knowledge of the relationships among soil macrofauna, soil quality and tree species in a smallholder agroforestry system of western Honduras, Geoderma, 189-190, 186-198.

Porwal, A. González-Álvarez, I. Markwitz, V., McCuaig, T.C. \& Mamuse, A. (2010). Weights-of-evidence and logistic regression modeling of magmatic nickel sulfide prospectivity in the Yilgarn Craton, Western Australia. Ore Geology Reviews, 38(3), 184-196.

Rubiano, J. \& Soto, V. (2009). Geographical extrapolation domain analysis: scaling up watershed management research projects, a toolkit to guide implementation. CPWF Working Paper 04. Colombo, Sri Lanka: The CGIAR Challenge Program on Water and Food.

Sawatzky, D., Raines, G. F. \& Bonham-Carter, G.F. (2009). SpatialData Modeller. Recuperado de http://www.ige.unicamp.br/sdm/ArcSDM93/source/ReadMe_ArcSDM2009.pdf.

Spiegelhalter, D. J. (2009). Incorporating Bayesian ideas into health-care evaluation. Statistical Science, 19, 156-74.

Terán Moreno, D. C. y Vidal, J.A. (2013). Sistemas Agroforestales. Universidad Nacional Abierta y a Distancia. Escuela de Ciencias Agrícolas, Pecuarias y del Medio Ambiente. 273p. Recuperado de: http://datateca.unad.edu.co/contenidos/201617/Guias_y_ rubricas_2013-II/Sistemas_Agroforestales_2013-2.pdf

Uchida, H. \& Nelson, A. (2010). Agglomeration index: Towards a new measure of urban concentration, Working paper // World Institute for Development Economics Research, No. 2010, 29, ISBN 978-92-9230-264-1

Recepción: 9 de junio de 2014

Evaluación: 13 de septiembre de 2014

Aprobación: 10 de octubre de 2014

218 Jorge Eliecer Rubiano Mejía, Mauricio Rincón Romero, Fabio Alexander Castro Llanos 